\title{
Internet of Things: Smart Things
}

\author{
Somayya Madakam
}

\begin{abstract}
Research study on the Internet of Things and Smart Things has been going on for more than a decade and reaches back to Mark Weiser's original dream of ubiquitous computing. Bruce Sterling recently popularized the idea of Smart Objects and the IoT. Smart Things is another paradigm shift in IT world. Smart Things are the things that are having embedding smartness or intelligence, identification, automation, monitoring and controlling calibre. Smart Things are assisting human life a lot, nowadays without their applications life is becoming cumbersome. This paper exhibits systematically on Internet, Things, and then explores on Internet of Things and finally Smart Things from researchers', and corporate's perspective. Moreover, this article focuses on the state of Smart Things and its applications. This in turn would help the new researchers, who want to do research in this IoT domain.
\end{abstract}

Index Terms - Internet, things, Internet of Things, IoT, smart things, Pachube, Nabaztag.

\section{INTRODUCTION}

\section{A. Internet}

The Internet is a powerful global communication medium that provides instantaneous information across geographical, cultural, language, and time spheres [1]. Internet is a global system of interconnected computer networks that use the standard Internet Protocol suite (TCP/IP: IPv4/IPv6) to serve billions of users worldwide. Internet is a network of networks that consists of millions of private, public, academic, research business, and government networks, of local to global scope, that are linked by a broad array of electronic, wireless and wired networking technologies [2].The Internet carries an extensive range of information resources and services, such as the inter-linked hypertext documents, now with the latest HTML5 of the world wide web and the infrastructure to support email. The internet brought marvellous changes into our daily life without leaving any field like day to day personal work, health, education, research, humanity, education, science, entertainment, communication, manufacturing, tourism, business, service, government sectors and so on. More than 180 countries are linked into exchanges of data, information, news and opinions. According to Internet World statistics, as of December 31, 2011 there was an estimated of 226, 72, 33, 742 Internet users at global level. This represents almost $32.7 \%$ of the total world's population. Even in the future Internet is going into space through Cisco's IRIS (Internet Routing in Space) program. This is the base for Smart Things concept.

Manuscript received August 20, 2014; revised June 12, 2015.

Somayya Madakam is with Information Technology Applications Group at National Institute of Industrial Engineering (NITIE), Mumbai, India (e-mail: somu4smart@gmail.com).

\section{B. Things}

Thing can be defined as an entity, an idea, a quality perceived, or thought to have its own existence in the world. Things are also often interchanged with the word "Objects". When we are talking about things, they could be both Living Things and Non-Living Things. Things, in this context, can be people, animals, plants, birds, servers, applications, shampoo bottles, cars, steering wheels, coffee machines, electronic devices, park benches or just about any other random item that comes to our mind, even which could be vicinity dust also. Everyday objects include not only electronic devices we encounter but also use daily, and technologically advanced products such as equipment and electronic gadgets, but "things" that we do not do normally think of as electronic at all - such as food, clothing, and furniture, materials, parts, merchandise and specialized items, landmarks, monuments and works of art and all the miscellany of commerce, culture and sophistication [3]. Once something has a unique identifier, it can be tagged, assigned a Uniform Resource Identifier (URI) and monitored over a network, automated other things and even talk too. Furthermore, one of the most used technologies today to connect objects, RFID, is not new idea, having been discovered by the Scottish physicist Robert Alexander Watson-Watt in year 1935 and used during II World War. Things are another side of the Smart Things coin.

\section{INTERNET OF THINGS}

Internet of Things is defined as "An open and comprehensive network of intelligent objects that have the capacity to auto-organize, share information, data and resources, reacting and acting in face situations and changes in the environment". Internet of Things is one of the last advances in Information and Communication Technologies, providing global connectivity and management of sensors, devices, users and information [4]. Imagining the Internet of Things (IoT) being used to track objects like a can of cola or a box of cereal from sites of production to sites of consumption is perhaps not too difficult to imagine. However, there is a movement under way to add almost every imaginable physical object into the Internet of Things. In New Zealand, for example, all cows might had IP addresses embedded in RFID chips implanted into their heads by 2011 [5]. Furthermore, objects are increasingly able to not just be characterized by a unique identifier, but also to transmit location, automate the things, monitor and context-sensitive datum. The Internet of Things refers to the coding and networking of everyday objects and the things to render them individually machine readable and traceable on the Internet [6]-[11]. Much existing content in the Internet of Things has been created through coded RFID tags and IP addresses linked into an Electronic 
Product Code network [12]. Currently, there are 9 billion interconnected devices and it is expected to reach 24 billion devices by 2020. US National Intelligence Counsel foresees that "by 2025 Internet nodes may reside in everyday things-food packages, furniture, paper documents and more" [3]. Internet of Things (IoT) describes a world where just about anything can be connected and communicates in an intelligent fashion that ever before.

\section{SMART THINGS}

\section{A. Evolutions}

Smart Things make our world smarter. Smart Things are a group of devices which can be monitored and controlled via a hub device (central processors) and web services. Smart Things adding support for popular connected products such as the Belkin WeMo family of devices, Philips Hue color-changing bulbs, and the Sonos home music system. The idea of smart objects and the IoT was recently popularized [13]. As with those products, Smart Things users will now be able to control and automate today's additions directly through the Smart Things applications. The world of smart fridges, smart washing machines, smart TV, other home appliances, smart shoes and smart phones is already in use, but the practice of user experience design for Internet of Things is still fairly new concept. Smart Things are one of the latest technology launched by innovators of Kickstarter. Design companies like IDEO and frog design are frequently asked to design products that unify software interaction, device design and service design - which are all the key components of Internet of Things. The current transition of the global Internet to IPv6 will provide a virtually unlimited number of public IP addresses able to provide bidirectional and symmetric (Machine-Machine: M2M) access to billions of Smart Things. Interaction and integration of Internet of Things in the global Internet are IPv6 integration, global interoperability, IoT - Cloud integration. In other words, how to bridge billion of Smart Things globally, while respecting their specific constraints. Weaving Smart Things, enterprises, and people leads to innovation in products, services and new business models in the fourth coming years.

Smart Things are autonomous physical or digital objects augmented with sensing, processing, acting and network capabilities. Adding smartness to everyday things in our world, so that our life can be more amazing. Smart Things places the world of linked things at your fingertips. Smart Things are more intelligent, convenient, secure, safe and efficient. These make it easy to connect the things in our physical world to the internet for automate, monitor, control and have fun with them from anywhere, at any time through any network by any one. This smartness can be done by different embedded Internet of Things technologies like RFID, EPC, barcode, IPv4 / IPv6, sensors, actuators, GIS, GPS, Wi-Fi, Bluetooth, ZigBee, NFC, ambient intelligence, Web 3.0 and telemedicine. We call smart any physical object connected to the web with some sensing capabilities. Its main capabilities are 1) Detect users and the social connections between them 2) Access user's data 3) Infer social context according to user's network topology, preferences and features 4) Infer social goals according to the social context and the user model 5) Coordinate their behaviour 6) Provide a context driven output [14]. Smart Devices are characterized by the ability to execute multiple, possibly concurrent, applications, supporting different degree of mobility and customisation and by supporting intermittent remote service and operating according to local resource constraints [15]. Smart Devices tend to be owned, operated, and configured and under the control of individual human users, For Example: personal computers, smart phones, cameras, game consoles, set up boxes and other computer peripherals, such as printers, mouse, external disk drives. In authors prediction IoT as a composition of smart objects that can understand and react to their environments. Based on practical experimentation and prototyping, smart objects are classified into Activity-aware objects, Policy-aware objects and Process-aware objects [16]. 1) Activity-aware objects: Amount of work performed to convert input into output 2) Policy-aware objects: The basic protocols issued by government or system administrator 3) Process-aware objects: A series of actions, motions, or occurrences. They also identified the smart-object design space as a space of three dimensions: awareness, representation \& interaction [3].

The Smart Things concept has four logical architectural layers 1) which connect to the Smart Things Hub or in some cases directly to the Cloud 2) which acts as a gateway for getting events and messages to or from the Cloud 3) which provides the abstraction and intelligence layers described above, as well as the web services that support the presentation layer 4) which provides the presentation layer for smart things in the form of mobile applications and our web IDE. Within the Smart Things-Cloud, however there are also four logical "Layers" of the architecture as well 1) Connectivity: Which is responsible for maintaining persistent connectivity to Smart Things Hubs, Smart Things Mobile application 2) Event processing and routing: This layer routes events from hubs or devices to smart applications that are subscribed to specific devices or events 3) Application: This layer provides the data access layer for data about accounts, users, and devices and is responsible for the execution of smart applications 4) Web Services: This layer provides the web services or Application Programmatic Interface (API) layer that supports both the mobile applications as well as developers who want to integrate from an external system using the Smart Things APIs.

\section{B. Concepts}

One of the buzzwords in the arena of Internet of Things applications is Smart Things. These applications can be found in our daily life right from the morning wake up to till the late go to the bed. Even in small villages, these applications are penetrated in the form of Smart Phones. Most of us think about "being connected" in terms of electronic devices such as servers, computers, tablets, telephones, smart phones and physical devices shoe, brush, bed, curtain and table. Smart Things describes a world where just about anything can be connected and communicates in an intelligent fashion that ever before. In other words, with the smart things, the physical world is becoming one big information system and Big Data Analytics (BDA) is helping in analysis for the better decisions. 
Although the concept wasn't named until 1999, the Internet of Things has been in development for decades. The first Internet appliance was a coke machine at Carnegie Melon University in the early 1980s. Programmers working several floors above the vending machine wrote a server program that tracked how long it had been since a storage column in the machine had been empty. The programmers could connect to the machine over the Internet, check the status of the machine and determine whether or not there would be a cold drink awaiting them, should they decide to make the trip down to the machine. Gradually importance of Smart Things under the umbrella of Internet of Things is grown up. There are various predictions about smart devices or things connecting to the internet based on different surveys. A report entitled 'Internet of Things' has predicted that there could be up to 16 billion connected devices by the year 2020 with an average of nearly 6 devices for every person on the planet. But IMS Research forecasts that we will have 22 billion Internet connected devices by the year 2020 (see Fig. 1 - Fig. 3).

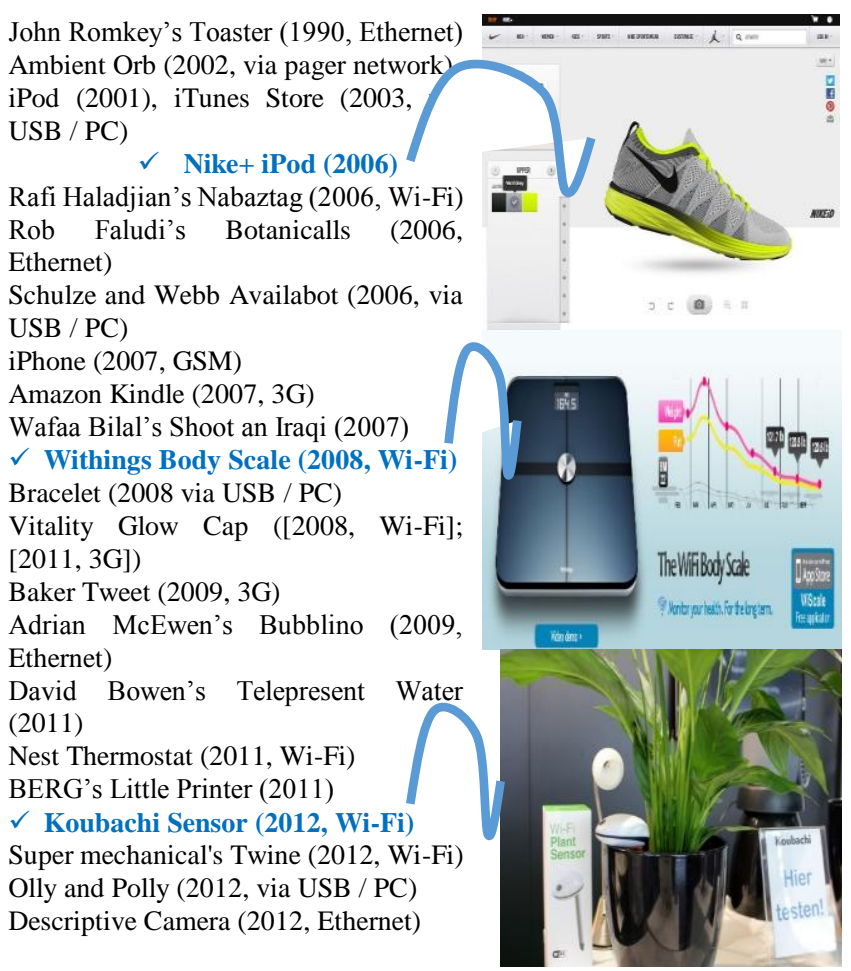

Fig. 1. Things connected through various IoT technologies.

\section{APPLICATIONS}

"It makes me so happy getting a short message when the kids get off the school bus so I know they got home safely" Unanimous One of the foremost applications of the Smart Things applications is focused on revolutionizing the Intelligent Homes or Home Automation industry. Home Automation has been a dream for decades with the idea of smart homes that would do lots of things automatically, predict our needs, and even talk to us. 1) Sterling coined first the term spime to describe a new group of space-time, location-aware, environment-aware, self-logging, self-documenting, uniquely identified objects that provide a lot of data about themselves and their environment. According to Sterling, one could track the entire existence of an object, from the time before it was made, through its manufacture, its ownership history, its physical location, to its eventual obsolescence and breaking-down back into raw material to be used for new instantiations of objects 2) The Hydra [17] middleware allows developers to include heterogeneous physical sensing devices into their applications by offering easy-to-use web service interfaces for controlling any type of physical sensor devices irrespective of its network technology. It also incorporates means for device and service discovery, semantic model driven architecture and security 3) NIKE+, 2006 a GPS chip fits into the insole of certain NIKE shoes, sending data to an application on your iPod or iPhone that allows you to track your running performances, set personal training goals and more. NIKE ID, 2009 - this online service allows customers to modify the colors and materials of certain NIKE shoes to their liking and then have them delivered to their door. The first applications of the IoT was in business processes such as the remote maintenance of industrial machinery, supply-chain optimization and security, and infrastructure management; but the technology went later saw its way into consumer devices. Some Smart Things services are 1) Thing speak 2) Twitter 3) Yaler 4) Pachube.

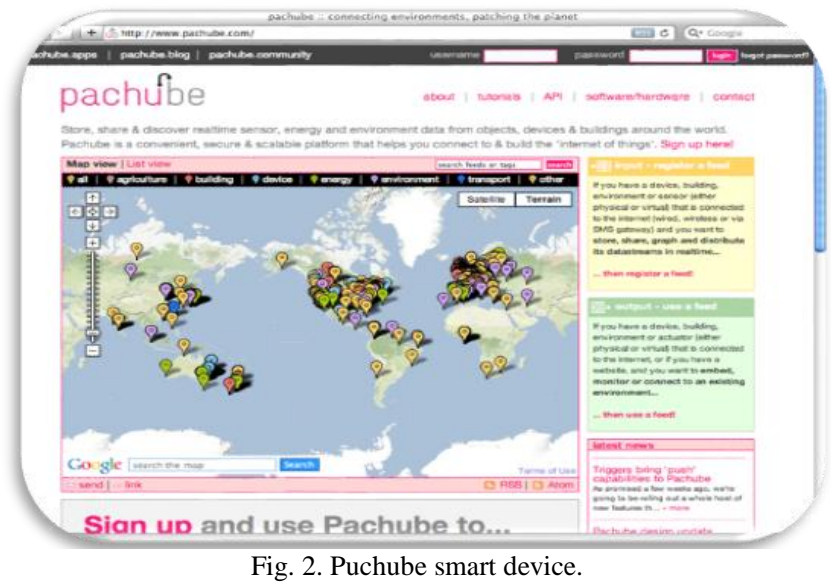

Pachube makes very simple to build applications, products and services that bridge physical and virtual worlds. With a fast advance cycle and a sophisticated growth path people have used Pachube to build sensor-logging systems, distant monitoring applications integrate building management systems, develop geographically tracking systems, generate mashups and networked objects and a whole host of other things. This is one of the best smart thing applications of Internet of Things. Pachube is used to store and reuse sensor measurements. Accessed dated on 21/ 07 /2014 from URL (http://www.haque.co.uk/pachube.php).

\section{A. Nabaztag}

Nabaztag ( $23 \mathrm{~cm}$ in height \& weighs $418 \mathrm{~g}$ ) is a Wi-Fi enabled ambient electronic device in the shape of a rabbit, invented by Rafi Haladjian and Olivier Mével. Manufactured by the Violet Company in the year 2005. First connect the rabbit, then connect everything else. That was the simple business belief that keeps Rafi Haladjian moving his charming little digital rabbit (Nabaztag) through the world, hooking it up to lamps and books and more. The rabbit created, is the starting point of French businessperson Haladjian's company Violet. Violet has a simple business mission that is nothing but "connect objects". Nabaztag is the 
Smart Thing, and it is designed to do more than we can imagine. It is shaped a little like a cream puff with bugs, bunny ears, due to its ability to connect to the internet through Wi-Fi connectivity, Nabaztag is able to tell us weather forecast, stock market report, news headlines, alarm clock, e-mail alerts, RSS-Feeds and MP3-Streams.This loveable rabbit communicates not just through his voice but through flashing lights and subtle movements, such as gestures with its ears. Accessed on dated on 21/7/2014 from the URL (http://genevalunch.com/2008/02/07/technology-is-a-smart-s mart-rabbit-touched-by-love/).

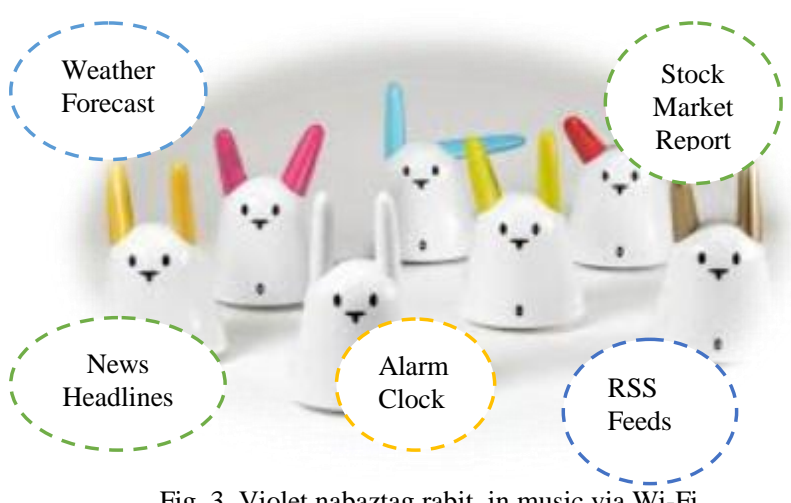

Fig. 3. Violet nabaztag rabit in music via Wi-Fi.

\section{CONCLUSION}

The Internet of Things consist of Smart Things. Smart Things are playing an active role in our everyday life, and these applications are fabulous and countless. They are not only into our domestic applications, but also into business and industrial applications comprising of manufacturing, chemical, productions, medical, pharmaceutical, Research \& Development. In addition the applications are spreading into other sectors like government, education, mining, geo-spatial and disaster management. The best part of Smart Things is that they are bringing the quality of life to human beings, operational efficiency and handles the situations where human being intervention is not at all possible. In the future, we need to focus more on Smart Things in terms of development, deployment, architectural, global level standardization, and ethical issues. We also need to concentrate on network communications protocols, as much trillions of smart devices or objects are connecting to the virtual world. Security is one more aspect, when things are communicate among themselves, which are not having intelligence power except via AI. Let us develop useful, harmonious Smart Things for easy, happy and secured life.

\section{REFERENCES}

[1] S.-I. Hou, S.-A. R. Charlery, and K. Roberson, "Systematic literature review of Internet interventions across health behaviours," Health Psychology and Behavioral Medicine: Open Access Journal, vol. 2 , no. 1, pp. 455-481, 2014.

[2] G. Nunberg, "The advent of the Internet," 2012.

[3] E. A. Kosmatos, N. D. Tselikas, and A. C. Boucouvalas, "Integrating RFIDs and smart objects into a unified Internet of things architecture," Advances in Internet of Things, vol. 1, pp. 5-12, 2011.

[4] J. Antonio et al., "Drugs interaction checker based on IoT," Internet of Things (IOT), pp. 1-8, IEEE, 2010.

[5] E. Wasserman, "Riding herd: RFID tracks live-stock," 2009.

[6] E. Biddlecombe, "UN predicts 'internet of things'," July 6, 2009.

[7] D. Butler, "2020 computing: Everything, everywhere," Nature, vol. 440, no. 7083, pp. 402-409, 2006.

[8] S. Dodson, "The net shapes up to get physical," Guardian, 2008.

[9] N. Gershenfeld, R. Krikorian, and D. Cohen, "The Internet of things," Scientific American, October, 2004.

[10] R. Lombreglia, "The Internet of things," Boston Globe, pp. 76-83, 2005.

[11] A. Reinhardt, "A machine-to-machine Internet of things," 2004.

[12] G. Mark, and H. Haarstad, "Transparency and development: Ethical consumption and economic development through Web 2.0 and the Internet of things," Information Technologies \& International, 2011.

[13] B. Sterling, Shaping Things, Cambridge: Massachusetts Institute of Technology Press, 2005.

[14] G. Biamino, "Semantic model for socially aware objects, advances in Internet of things," vol. 2, pp. 47-55, 2012.

[15] S. Poslad, Ubiquitous Computing: Smart Devices, Environments and Interactions, John Wiley \& Sons, Ltd., p. 75, 2009.

[16] G. Kortuem, F. Kawsar, D. Fitton, and V. Sundramoorthy, "Smart objects as building blocks for the Internet of things," IEEE Internet Computing, vol. 14, no. 1, pp. 44-51, 2010.

[17] Hydra project. (2010). [Online]. Available: http://www.hydramiddleware.eulUniversal

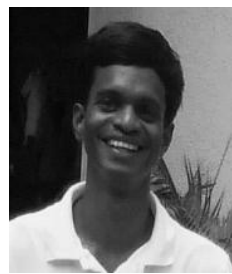

Somayya Madakam is the fellow student of $3^{\text {rd }}$ year (2014-2015) at National Institute of Industrial Engineering (NITIE), Mumbai (India). He worked as an systems analyst cum programmer in Tata Institute of Social Sciences (TISS), Mumbai (India) for six years. His experience includes system design, computers network administration, website management, IT-business process, e-mail service solutions and software troubleshooting. He received his MBA with dual specialization of IT management along with HR management from Andhra University. Prior to this he graduated B.Tech (computer science \& systems engineering) from Andhra University, Vizag (India). 